\title{
Design and Synthesis of Novel Chalcone-Phenylpyranone Derivatives as Estrogen Receptor Modulators ${ }^{\dagger}$
}

\author{
Pritam N. Dube ${ }^{1, *}$, Yogita B. Thombare ${ }^{2}$ and Vivekanand A. Chatpalliwar ${ }^{1}$ \\ 1 Department of Pharmaceutical Chemistry, SNJB's SSD Jain College of Pharmacy, Neminagar, Chandwad, \\ Nashik 423101, Maharashtra, India; vchatpalliwar@yahoo.co.in \\ 2 Department of Pharmaceutical Chemistry, MET Institute of Pharmacy, Bhujbal Knowledge City, Adgaon, \\ Nashik 422003, Maharashtra, India; yogitathombare11@gmail.com \\ * Correspondence: pritamdube@gmail.com \\ + Presented at the 22nd International Electronic Conference on Synthetic Organic Chemistry, 15 \\ November-15 December 2018; Available Online: https://sciforum.net/conference/ecsoc-22. \\ Published: 23 November 2018
}

\begin{abstract}
Selective estrogen receptor modulators (SERMs) are a class of drugs that act on the estrogen receptor (ER). SERMs are used for treatment and reduction of risk of breast cancer. Herewith we had designed, synthesized, and evaluated chalcone-phenylpyran-2-one derivatives bearing a $\mathrm{N}, \mathrm{N}$-dimethyl ethylamine side chain for their anti-breast cancer activity on MCF-7 and Zr-75-1 cell lines in-vitro. The pharmacological data indicated that most of tested compounds showed moderate to significant cytotoxicity and high selectivity toward the estrogen receptor. The Structure activity relationaship analyses indicated that compounds $5 \mathbf{f}$ with 2,6-dichloro substitution was more effective. Docking study was performed to predict binding orientation towards the estrogen receptor- $\alpha$.
\end{abstract}

Keywords: breast cancer; chalcone; docking; MCF-7; phenylpyranone; SERM

\section{Introduction}

Breast cancer mortality has declined by $24 \%$ from 1990-2000, likely due to increases in the use of both mammography screenings (followed by surgery) and adjuvant therapy, including chemotherapy and antihormonal therapy. Worldwide, it is anticipated that in the coming decade, five million women will be affected by breast cancer. Clearly, further advances in the development of treatments, particularly ones with fewer undesirable side-effects, are necessary [1,2].

Endocrine therapy alone-most notably Tamoxifen, a selective estrogen receptor modulator (SERM) that blocks estrogen action in breast cancer-is estimated to account for a $9.8 \%$ (median value) decrease in breast cancer mortality [3].

Phytoestrogens are plant derived substances that are structurally and functionally similar to estrogens and are found in many foods. Mainly there are three classes of phytoestrogens-isoflavones, coumestans, and lignans. Epidemiological data indicate that Asiatic societies which consume phytoestrogen-rich diet have a lower risk of so called "Western diseases" such as breast and prostate cancer, osteoporosis, and cardiovascular diseases [1,3,4].

Chalcones (1,3-diaryl-2-propen-1-ones) are a class of compounds consisting of two aryl rings linked by an a,b-unsaturated ketone moiety as shown in Figure 1. Chalcone moieties are common substructures in numerous natural products belonging to the flavonoid family. Derivatives of chalcone are versatile as pharmaceutically active compounds and have been shown to display many desirable properties for human diseases, including anticancer, anti-HIV, antimalarial, antioxidant, 
anti-inflammatory, and anti-allergic activities. Published data showed that chalcone compounds possess strongantiproliferative activities against both primary cells and established cell lines.

We synthesized 6heteroarylchalcone compounds and examined their antiproliferative activity against breast cancer cell lines. We show here for the first time that heteroarylchalcones can be effective therapeutics against human breast cancer with relatively low side effects on non-cancer cells $[4,5]$.

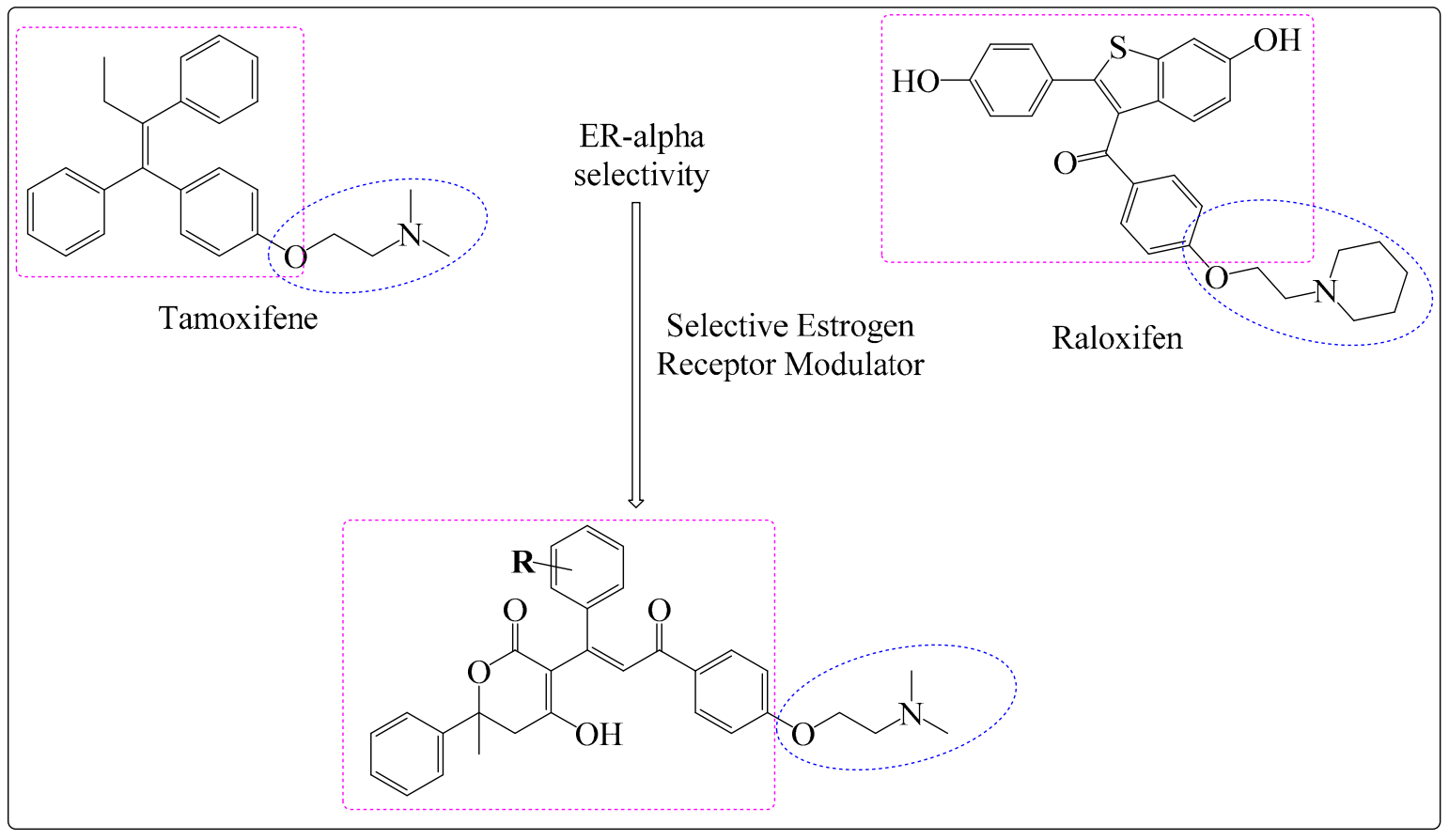

Figure 1. Pharmacophore of designed compounds.

\section{Material and Methods}

\subsection{Chemistry}

Melting points were recorded in open capillaries with an electrical melting point apparatus and were uncorrected. IR spectra of all synthesized compound in $\mathrm{KBr}$ were recorded utilizing a (JASCO FT-IR 4000) spectrophotometer. ${ }^{1} \mathrm{H}$ and ${ }^{13} \mathrm{C}$ NMR spectra were recorded on a BrukerAvance, Billerica, MA, USA (400 MHz) spectrometer in $\mathrm{CDCl}_{3}$ solutions, with TMS as an internal reference. Mass spectra were recorded on a Varian Inc., Palo Alto, CA, USA, 410 Prostar Binary LC with 500 MS IT PDA Detectors. All the reagents and solvents used were of analytical grade.

\subsubsection{Synthesis of 4-Hydroxy-6-methyl-6-phenyl-5,6-dihydro-2H-pyran-2-one (b)}

As shown in step 1 (Scheme 1), a mixture of acetophenone (a) and ethyl 3-oxobutanoate, $\mathrm{NaH}$, and $\mathrm{n}$-BuLi were stirred overnight at $0{ }^{\circ} \mathrm{C}$. The resultant solution was worked up into ice, and washed with water. The precipitate was recrystallized from absolute ethanol. 
Step 1<smiles>CCOC(=O)CC(C)(Br)C(C)Br</smiles><smiles>CC1(c2ccccc2)CC(O)=CC(=O)O1</smiles>

Step 2<smiles>[R]=CC=CC=O</smiles>

substituted aldehyde (1)<smiles>CC(=O)c1ccc(O)cc1</smiles>

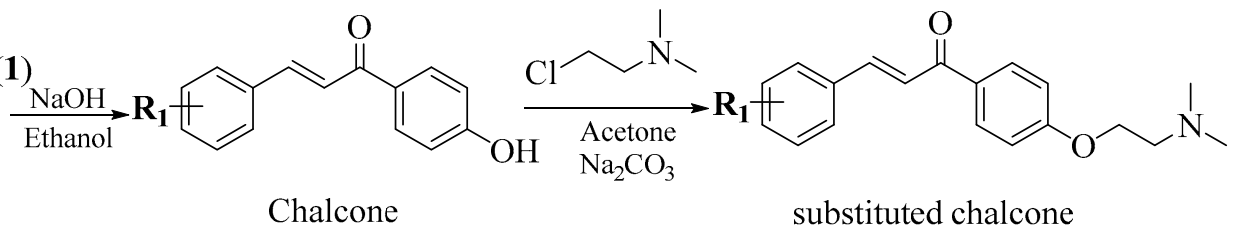

4-hydroxy acetophenone (2)

$(\mathbf{3 a}-\mathbf{3 f})$

$(4 a-4 f)$<smiles>CC1(c2ccccc2)CC(O)=CC(=O)O1</smiles><smiles>[R17]c1ccc(C(=CC(=O)c2ccc(OCCN(C)C)cc2)C2=C(O)CC(C)(c3ccccc3)OC2=O)cc1</smiles>

$5 \mathbf{a}-\mathbf{5 f}$

Scheme 1. Synthesis of designed compounds (5a-f).

\subsubsection{Synthesis of Substituted p-HydroxyChalcone (3)}

An equimolar mixture of 4-hydroxy acetophenone, substituted benzaldehydes, and $\mathrm{KOH}(2$ mmol) was stirred in PEG-400 (15 mL) at $40{ }^{\circ} \mathrm{C}$ for $2-3 \mathrm{~h}$. After the completion of the reaction (monitored by TLC), the crude mixture was worked up in ice-cold water (100 mL). The resultant product was separated out and recrystallized from absolute ethanol $[5,6]$.

\subsubsection{Synthesis of Substituted [(4-(2-(Dimethylamino)ethoxy)] Chalcone}

A mixture of substituted chalcone $(0.625 \mathrm{mmol})$, anhydrous $\mathrm{K}_{2} \mathrm{CO}_{3}(3.12 \mathrm{mmol})$, 2-chloro- $\mathrm{N}, \mathrm{N}$-dimethylethanamine $(0.93 \mathrm{mmol})$, and dry acetone $(10 \mathrm{~mL})$ was refluxed for $24 \mathrm{~h}$. $\mathrm{K}_{2} \mathrm{CO}_{3}$ was filtered off, and acetone was distilled out. The residue was diluted with water and extracted with ethyl acetate. The organic layer was washed with water and brine, and it was dried over anhydrous $\mathrm{Na}_{2} \mathrm{SO}_{4}$. The precipitate was recrystallized from absolute ethanol $[6,7]$.

\subsubsection{Synthesis of Target Compounds (5a-f)}

A mixture of substituted [(4-(2-(dimethylamino)ethoxy)] chalcone $(0.497 \mathrm{mmol})$, compound $\mathbf{b}$ $(0.204 \mathrm{mmol})$, imidazolidine catalyst $(10 \mathrm{mmol})$, andTHF $(10 \mathrm{~mL})$ was stirred for $36 \mathrm{~h}$. The residue was diluted with water and extracted with ethyl acetate. The organic layer was washed with water and brine, and it was dried over anhydrous $\mathrm{Na}_{2} \mathrm{SO}_{4}$. The precipitate was recrystallized from absolute ethanol $[7,8]$. 
3-(3-(4-(2-(Dimethylamino)ethoxy)phenyl)-3-oxo-1-phenylprop-1-en-1-yl)-4-hydroxy-6-methyl-6-ph enyl-5,6-dihydro-2H-pyran-2-one (5a)

\% Yield:66.24; MW: 497.59; MF: $\mathrm{C}_{31} \mathrm{H}_{31} \mathrm{NO}_{5}$; MP: 142-144 ${ }^{\circ} \mathrm{C}$; IR (KBr): 677 (Ar-H), 1191 (C-O), 1339 (C-N), $1538(\mathrm{C}=\mathrm{C}), 1722(\mathrm{C}=\mathrm{O}) \mathrm{cm}^{-1}$; $1 \mathrm{H}$ NMR (DMSO, $\left.400 \mathrm{MHz}\right): \delta=8.4(\mathrm{~s}, 1 \mathrm{H}, \mathrm{OH}), 7.8-7.4(\mathrm{~m}, 14 \mathrm{H}$, Ar-H), $7.1(\mathrm{~s}, 1 \mathrm{H}, \mathrm{CH}), 4.1\left(\mathrm{t}, 2 \mathrm{H}, \mathrm{CH}_{2}\right), 2.9\left(\mathrm{t}, 2 \mathrm{H}, \mathrm{CH}_{2}\right), 2.3,2.5\left(\mathrm{~s}, 2 \mathrm{H}, \mathrm{CH}_{2}\right.$ of pyranone), $2.2(\mathrm{~s}, 6 \mathrm{H}$, $\mathrm{CH}_{3}$ of diamine), 1.8 (s, 3H, $\mathrm{CH}_{3}$ of pyranone); ${ }^{13} \mathrm{C}$ NMR (DMSO, $\left.100 \mathrm{MHz}\right): \delta=186.8(\mathrm{C}=\mathrm{O}), 179.2$ $(\mathrm{OH}), 164.5$ (Ar-C-O), $162.1(\mathrm{C}=\mathrm{O}), 154.7$ (Phenyl-pyranone), 142.4, 140.6 (Ar-propene), 130.2, 129.8, 129.7, 129.2, 128.4, 127.8-127.4 (Ar-C), 68.9 (C pyranone), $57.3\left(\mathrm{CH}_{2}-\mathrm{N}\right), 51.4\left(\mathrm{CH}_{2}-\mathrm{O}\right), 48.9,48.8\left(\mathrm{CH}_{3}-\mathrm{N}\right)$, $31.3\left(\mathrm{CH}_{3}\right) ; \mathrm{MS}: \mathrm{m} / \mathrm{z}=498.4[\mathrm{M}+1]$.

3-(1-(4-Chlorophenyl)-3-(4-(2-(dimethylamino)ethoxy)phenyl)-3-oxoprop-1-en-1-yl)-4-hydroxy-6-m ethyl-6-phenyl-5,6-dihydro-2H-pyran-2-one (5b)

\% Yield: 71.30; MW: 532.03; MF: $\mathrm{C}_{31} \mathrm{H}_{30} \mathrm{ClNO}_{5}$; MP: 170-172 ${ }^{\circ} \mathrm{C}$; IR (KBr): 681, 678 (Ar-H), 1194 (C-O), $1329(\mathrm{C}-\mathrm{N}), 1529(\mathrm{C}=\mathrm{C}), 1725(\mathrm{C}=\mathrm{O}) \mathrm{cm}^{-1}$; ${ }^{1} \mathrm{H}$ NMR (DMSO, $\left.400 \mathrm{MHz}\right): \delta=8.7(\mathrm{~s}, 1 \mathrm{H}, \mathrm{OH}), 7.9-7.4(\mathrm{~m}$, 13H, Ar-H), $7.0(\mathrm{~s}, 1 \mathrm{H}, \mathrm{CH}), 4.2\left(\mathrm{t}, 2 \mathrm{H}, \mathrm{CH}_{2}\right), 2.9\left(\mathrm{t}, 2 \mathrm{H}, \mathrm{CH}_{2}\right), 2.4,2.5\left(\mathrm{~s}, 2 \mathrm{H}, \mathrm{CH}_{2}\right.$ of pyranone), 2.1 (s, $6 \mathrm{H}, \mathrm{CH}_{3}$ of diamine), 1.9 (s, $3 \mathrm{H}, \mathrm{CH}_{3}$ of pyranone); ${ }^{13} \mathrm{C}$ NMR (DMSO, $\left.100 \mathrm{MHz}\right): \delta=188.9(\mathrm{C}=\mathrm{O}), 179.4$ (OH), 168.6 (Ar-C-O), $163.0(\mathrm{C}=\mathrm{O}), 157.1$ (Phenyl-pyranone), 143.5, 142.8 (Ar-propene), 130.4, 130.2, 129.8, 129.5, 129.1, 128.3, 127.7-127.2 (Ar-C), $68.7\left(\mathrm{C}_{\text {pyranone }}\right), 57.2\left(\mathrm{CH}_{2}-\mathrm{N}\right), 51.7\left(\mathrm{CH}_{2}-\mathrm{O}\right), 48.8,48.7$ $\left(\mathrm{CH}_{3}-\mathrm{N}\right), 32.4\left(\mathrm{CH}_{3}\right) ; \mathrm{MS}: \mathrm{m} / \mathrm{z}=534.2[\mathrm{M}+2]$.

3-(3-(4-(2-(Dimethylamino)ethoxy)phenyl)-3-oxo-1-(p-tolyl)prop-1-en-1-yl)-4-hydroxy-6-methyl-6-p henyl-5,6-dihydro-2H-pyran-2-one (5c)

\% Yield: 54.82; MW: 511.62; MF: $\mathrm{C}_{32} \mathrm{H}_{33} \mathrm{NO}_{5}$; MP: 158-160 ${ }^{\circ} \mathrm{C}$; IR (KBr): 668 (Ar-H), 1190 (C-O), 1333 (C-N), $1540(\mathrm{C}=\mathrm{C}), 1723(\mathrm{C}=\mathrm{O}) \mathrm{cm}^{-1}$; ${ }^{1} \mathrm{H}$ NMR (DMSO, $\left.400 \mathrm{MHz}\right): \delta=8.1(\mathrm{~s}, 1 \mathrm{H}, \mathrm{OH}), 7.8-7.3(\mathrm{~m}, 13 \mathrm{H}$, Ar-H), $7.1(\mathrm{~s}, 1 \mathrm{H}, \mathrm{CH}), 4.2\left(\mathrm{t}, 2 \mathrm{H}, \mathrm{CH}_{2}\right), 2.9\left(\mathrm{t}, 2 \mathrm{H}, \mathrm{CH}_{2}\right), 2.5\left(\mathrm{~s}, 3 \mathrm{H}, \mathrm{CH}_{3}\right), 2.3,2.5\left(\mathrm{~s}, 2 \mathrm{H}, \mathrm{CH}_{2}\right.$ of pyranone), 2.2 (s, $6 \mathrm{H}, \mathrm{CH}_{3}$ of diamine), $1.8\left(\mathrm{~s}, 3 \mathrm{H}, \mathrm{CH}_{3}\right.$ of pyranone); ${ }^{13} \mathrm{C}$ NMR (DMSO, $100 \mathrm{MHz}$ ): $\delta=$ $182.5(\mathrm{C}=\mathrm{O}), 175.1(\mathrm{OH}), 166.6$ (Ar-C-O), $163.2(\mathrm{C}=\mathrm{O}), 151.8$ (Phenyl-pyranone), 143.0, 141.6 (Ar-propene), 131.3, 130.2, 129.8, 129.5, 128.8, 127.8-127.5 (Ar-C), 61.5 (C $\left(\mathrm{CH}_{2}-\mathrm{O}\right), 48.8,48.7\left(\mathrm{CH}_{3}-\mathrm{N}\right), 33.5,31.6\left(\mathrm{CH}_{3}\right) ; \mathrm{MS}: \mathrm{m} / \mathrm{z}=512.6[\mathrm{M}+1]$.

3-(3-(4-(2-(Dimethylamino)ethoxy)phenyl)-1-(4-methoxyphenyl)-3-oxoprop-1-en-1-yl)-4-hydroxy-6methyl-6-phenyl-5,6-dihydro-2H-pyran-2-one (5d)

\% Yield: 50.14; MW: 527.23; MF: $\mathrm{C}_{32} \mathrm{H}_{33} \mathrm{NO}_{6}$; MP: 180-182 ${ }^{\circ} \mathrm{C}$; IR (KBr): 680 (Ar-H), 1190 (C-O), 1335 (C-N), $1539(\mathrm{C}=\mathrm{C}), 1724(\mathrm{C}=\mathrm{O}) \mathrm{cm}^{-1}$; ${ }^{1} \mathrm{H}$ NMR (DMSO, $\left.400 \mathrm{MHz}\right): \delta=8.1(\mathrm{~s}, 1 \mathrm{H}, \mathrm{OH}), 7.8-7.4(\mathrm{~m}, 13 \mathrm{H}$, Ar-H), $7.2(\mathrm{~s}, 1 \mathrm{H}, \mathrm{CH}), 4.2\left(\mathrm{t}, 2 \mathrm{H}, \mathrm{CH}_{2}\right), 3.3\left(\mathrm{~s}, 3 \mathrm{H}, \mathrm{CH}_{3}-\mathrm{O}\right), 2.9\left(\mathrm{t}, 2 \mathrm{H}, \mathrm{CH}_{2}\right), 2.3,2.4\left(\mathrm{~s}, 2 \mathrm{H}, \mathrm{CH}_{2}\right.$ of pyranone), 2.1 (s, $6 \mathrm{H}, \mathrm{CH}_{3}$ of diamine), $1.8\left(\mathrm{~s}, 3 \mathrm{H}, \mathrm{CH}_{3}\right.$ of pyranone); ${ }^{13} \mathrm{C}$ NMR (DMSO, $\left.100 \mathrm{MHz}\right): \delta=$ $180.6(\mathrm{C}=\mathrm{O}), 177.3(\mathrm{OH}), 164.7(\mathrm{Ar}-\mathrm{C}-\mathrm{O}), 162.2(\mathrm{C}=\mathrm{O}), 159.7$ (Ar-OCH$), 153.5$ (Phenyl-pyranone),

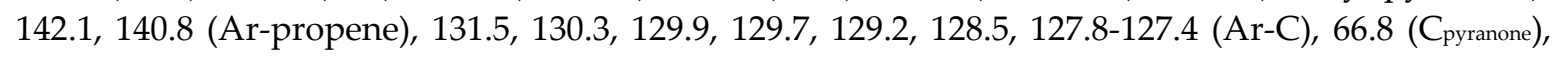
$57.7\left(\mathrm{CH}_{2}-\mathrm{N}\right), 55.1\left(\mathrm{OCH}_{3}\right), 52.0\left(\mathrm{CH}_{2}-\mathrm{O}\right), 49.1,48.9\left(\mathrm{CH}_{3}-\mathrm{N}\right), 31.2\left(\mathrm{CH}_{3}\right) ; \mathrm{MS}: \mathrm{m} / \mathrm{z}=528.2[\mathrm{M}+1]$.

3-(3-(4-(2-(Dimethylamino)ethoxy)phenyl)-3-oxo-1-(2,3,4-trimethoxyphenyl)prop-1-en-1-yl)-4-hydr oxy-6-methyl-6-phenyl-5,6-dihydro-2H-pyran-2-one (5e)

\% Yield: 68.62; MW: 587.67; MF: $\mathrm{C}_{34} \mathrm{H}_{37} \mathrm{NO}$; $\mathrm{MP}: 194-196{ }^{\circ} \mathrm{C}$; IR (KBr): 671 (Ar-H), 1188 (C-O), 1341 $(\mathrm{C}-\mathrm{N}), 1540(\mathrm{C}=\mathrm{C}), 1726(\mathrm{C}=\mathrm{O}) \mathrm{cm}^{-1}$; ${ }^{1} \mathrm{H}$ NMR (DMSO, $\left.400 \mathrm{MHz}\right): \delta=8.3(\mathrm{~s}, 1 \mathrm{H}, \mathrm{OH}), 7.9-7.4(\mathrm{~m}, 11 \mathrm{H}$, Ar-H), $7.0(\mathrm{~s}, 1 \mathrm{H}, \mathrm{CH}), 4.2\left(\mathrm{t}, 2 \mathrm{H}, \mathrm{CH}_{2}\right), 3.8-3.7\left(\mathrm{~s}, 9 \mathrm{H}, \mathrm{OCH}_{3}\right), 3.0\left(\mathrm{t}, 2 \mathrm{H}, \mathrm{CH}_{2}\right), 2.5,2.4\left(\mathrm{~s}, 2 \mathrm{H}, \mathrm{CH}_{2}\right.$ of pyranone), 2.1 (s, $6 \mathrm{H}, \mathrm{CH}_{3}$ of diamine), $1.8\left(\mathrm{~s}, 3 \mathrm{H}, \mathrm{CH}_{3}\right.$ of pyranone); ${ }^{13} \mathrm{C}$ NMR (DMSO, $\left.100 \mathrm{MHz}\right): \delta=$ $182.1(\mathrm{C}=\mathrm{O}), \quad 177.4 \quad(\mathrm{OH}), 164.4 \quad(\mathrm{Ar}-\mathrm{C}-\mathrm{O}), 162.8 \quad(\mathrm{C}=\mathrm{O}), \quad 161.1,159.7 \quad\left(\mathrm{Ar}-\mathrm{OCH}_{3}\right), 154.7$ (Phenyl-pyranone), 142.4, 140.6 (Ar-propene), 130.2, 129.8, 129.7, 129.2, 128.4, 127.8-127.4 (Ar-C),

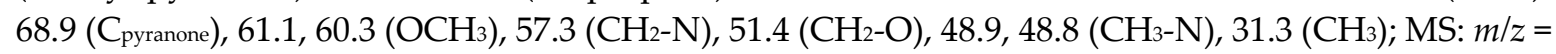
$588.6[\mathrm{M}+1]$. 
3-(1-(2,6-Dichlorophenyl)-3-(4-(2-(dimethylamino)ethoxy)phenyl)-3-oxoprop-1-en-1-yl)-4-hydroxy-6 -methyl-6-phenyl-5,6-dihydro-2H-pyran-2-one (5f)

\% Yield: 59.72; MW: 566.48; MF: $\mathrm{C}_{31} \mathrm{H}_{29} \mathrm{Cl}_{2} \mathrm{NO}_{5}$; MP: 170-172 ${ }^{\circ} \mathrm{C}$; IR (KBr): 682 (Ar-H), 1190 (C-O), $1327(\mathrm{C}-\mathrm{N}), 1529(\mathrm{C}=\mathrm{C}), 1725(\mathrm{C}=\mathrm{O}) \mathrm{cm}^{-1}$; ${ }^{1} \mathrm{H}$ NMR (DMSO, $\left.400 \mathrm{MHz}\right): \delta=8.6(\mathrm{~s}, 1 \mathrm{H}, \mathrm{OH}), 7.9-7.4(\mathrm{~m}$, 12H, Ar-H), $7.1(\mathrm{~s}, 1 \mathrm{H}, \mathrm{CH}), 4.3\left(\mathrm{t}, 2 \mathrm{H}, \mathrm{CH}_{2}\right), 2.9\left(\mathrm{t}, 2 \mathrm{H}, \mathrm{CH}_{2}\right), 2.5,2.4\left(\mathrm{~s}, 2 \mathrm{H}, \mathrm{CH}_{2}\right.$ of pyranone), 2.1 (s, $6 \mathrm{H}, \mathrm{CH}_{3}$ of diamine), 1.9 (s, 3H, $\mathrm{CH}_{3}$ of pyranone); ${ }^{13} \mathrm{C}$ NMR (DMSO, $100 \mathrm{MHz}$ ): $\delta=187.5(\mathrm{C}=\mathrm{O}), 179.8$ (OH), 168.8 (Ar-C-O), 164.1 (C=O), 157.3 (Phenyl-pyranone), 143.8, 142.4 (Ar-propene), 131.1, 130.8,

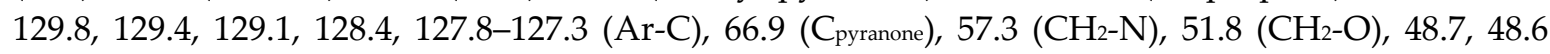
$\left(\mathrm{CH}_{3}-\mathrm{N}\right), 33.5\left(\mathrm{CH}_{3}\right) ; \mathrm{MS}: \mathrm{m} / \mathrm{z}=568.5[\mathrm{M}+2]$.

\subsection{In-Vitro Antitumor Activity}

In-vitro testing done using SRB assay protocols [14].Each drug was tested at 4 dose levels $(1 \times$ $10^{-7} \mathrm{M}, 1 \times 10^{-6} \mathrm{M}, 1 \times 10^{-5} \mathrm{M}$, and $1 \times 10^{-4} \mathrm{M}$, or $10,20,40$, and $80 \mu \mathrm{g} / \mathrm{mL}$ ). Appropriate positive controls were run in each experiment and each experiment was repeated thrice. Results were given in terms of GI50, TGI, and LC 50 values. The compounds were tested for their cytotoxic assay using MCF-7and ZR-75-1 breast cancer cell lines[8,9].

\section{Result and Discussion}

\subsection{Chemistry}

All the compounds were synthesized according to steps depicted in Scheme 1, and their structures were verified by IR, ${ }^{1} \mathrm{H}-\mathrm{NMR},{ }^{13} \mathrm{C}-\mathrm{NMR}$, and LC-MS spectroscopy. The first step in the synthetic route consisted of the cyclisation of acetophenone with ethyl 3-oxobutanoate in the presence of $\mathrm{n}$-BuLi and THF as solvent at $0{ }^{\circ} \mathrm{C}$ to give 4-hydroxy-6-methyl-6-phenyl-5,6-dihydro-2H-pyran-2-one (compound b) (Scheme 1). The second step consisted of the synthesis of chalcone $(\mathbf{3 a}-\mathbf{f})$. The etherification of chalcone was done at hydroxyl group by amino side chain, i.e., 2-chloro- $N, N$-dimethylethan-1-amine. The target compounds were synthesized by addition of compound $\mathbf{b}$ to $\mathbf{4 a}-\mathbf{f}$. Thesecompounds are shown in Table 1.

Table 1. In-vitro anticancer activity $(\mu \mathrm{g} / \mathrm{mL})$ of synthesized compounds (5a-f).

\begin{tabular}{|c|c|c|c|c|c|c|c|}
\hline \multirow{2}{*}{ Sr. No. } & \multirow{2}{*}{$\mathbf{R}$} & \multicolumn{3}{|c|}{ MCF-7 } & \multicolumn{3}{|c|}{ ZR-75-1 } \\
\hline & & $\mathrm{LC}_{50^{\mathrm{a}}}$ & TGI $^{b}$ & $\mathrm{GI}_{50^{\mathrm{c}}}^{\mathrm{c}}$ & $\mathrm{LC}_{50}$ & TGI & $\mathrm{GI}_{50}$ \\
\hline $5 a$ & & $>100$ & $>100$ & 88.5 & $>100$ & $>100$ & 78.6 \\
\hline $5 b$ & & $>100$ & $>100$ & 74.6 & $>100$ & $>100$ & 91.2 \\
\hline $5 c$ & & $>100$ & $>100$ & 52.2 & $>100$ & $>100$ & $>100$ \\
\hline $5 d$ & & $>100$ & $>100$ & 36.8 & $>100$ & $>100$ & 89.4 \\
\hline $5 e$ & & $>100$ & $>100$ & 68.3 & $>100$ & $>100$ & 71.9 \\
\hline
\end{tabular}




\begin{tabular}{lllllllll}
\hline TAM & - & 84.4 & 61.3 & $\mathbf{2 8 . 2}$ & $>100$ & $>100$ & 58.2 \\
ADR & - & 87.8 & 25.6 & $<0.1$ & $>100$ & $>100$ & $<0.1$ \\
& & 39.5 & 16.3 & $<10$ & $>100$ & $>100$ & $<0.1$ \\
\hline
\end{tabular}

Most potent compounds shown by bold text as compare to standard ${ }^{\text {TAM }}$ tamoxifen, ${ }^{A D R}$ Adriamycin. ${ }^{a}$ Compound concentration that produces $50 \%$ cytocidal effect. ${ }^{b}$ Compound concentration that

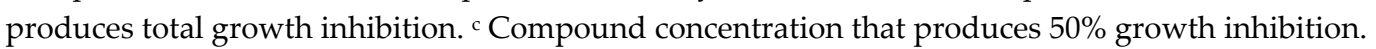

\subsection{In-VitroCytotoxic Assay}

The target compounds were evaluated for anticancer activity against estrogen receptor alpha positive (ER+) human breast cancer cell lines, i.e., MCF-7 and ZR-75-1. The in-vitro activity profile is shown in Table 1. The GI50 concentration for each compound was calculated with reference to a control sample, which represents the concentration that results in a $50 \%$ decrease in cell growth/proliferation after $48 \mathrm{~h}$ incubation in the presence of the drug. The total growth inhibition (TGI) is the concentration of test drug which signifies a cytostatic effect. The LC 50 is concentration of compound that produces the $50 \%$ cytotoxic effect. Tamoxifen and Adriamycin were used as reference.

The compound $\mathbf{5} \mathbf{f}$ showed most prominent cytotoxic activity against the MCF-7 breast cancer cell line. The structure activity indicates that the presence of hydrophobic group atpositions 2 and 6 at the substituted phenyl ring increases the activity, while substitution at the para position increases activity. The trimethoxy substitution also showed decrease in activity. The synthesized derivatives have the half potency to that of standard tamoxifen.

\subsection{Docking Analysis}

The docking study was performed online at Mcule.com using 1 Click docking on estrogen receptor alpha (PDB code: 1L2I). Binding orientation showed that the tertiary amine is important for hydrogen binding with Asp351 amino acid, while substitution with hydrophobic group at phenyl ring increases docking score, as shown in Figure 2.

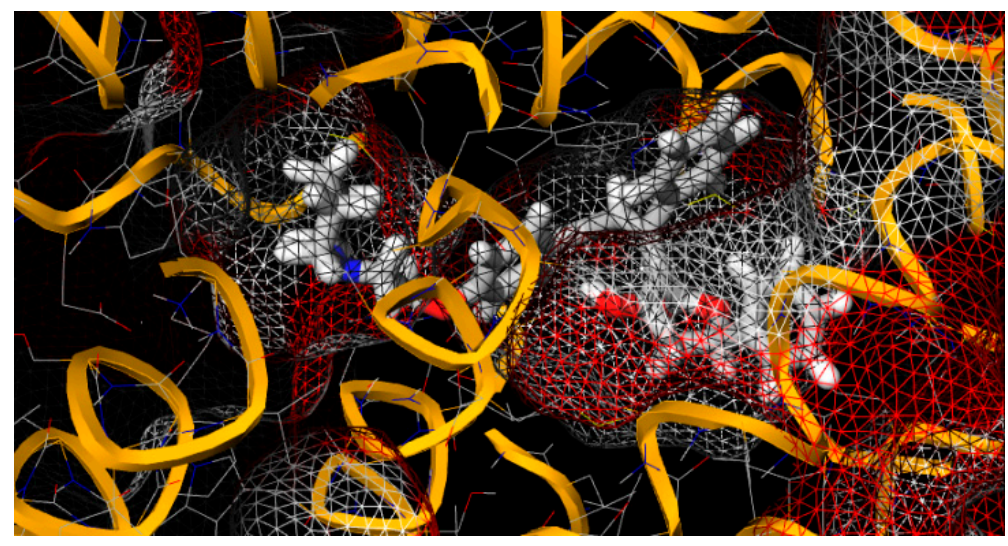

Figure 2. docking pose of compound $5 \mathbf{a}$.

\section{References}

1. Jensen, E.V.; Jordan, V.C. The estrogen receptor: A model for molecular medicine. Clin. Cancer Res. 2003, 9, 1980-1989.

2. Jordan, V.C.; Brodie, A.M. Development and evolution of therapies targeted to the estrogen receptor for the treatment and prevention of breast cancer. Steroids 2007, 72, 7-25.

3. Johnston, S.R.D.; Ford, H.; Ross, P. The Royal Marsden Hospital Hand Book of Cancer Chemotherapy; Brighton, D., Wood, M., Eds.; Elsevier Churchill Livingstone: London, UK; New York, NY, USA; Oxford, UK, 2005; pp. 1-17. 
4. Dube, P.N.; Waghmare, M.N.; Mokale, S.N. Synthesis, in Vitro, and in Vivo Biological Evaluation and Molecular Docking Analysis of Novel 3-(3-oxo-substitutedphenyl-3-)4-(2-(piperidinyl)ethoxy)phenyl)propyl)-2H-chromen-2-one Derivatives as Anti-breast Cancer Agents. Chem. Biol. Drug Des. 2016, 87, 608-617.

5. Sashidhara, K.V.; Kumar, A.; Kumar, M.; Sarkar, J.; Sinha, S. Synthesis and in vitro evaluation of novel coumarin-chalcone hybrids as potential anticancer agents. Bioorg. Med. Chem. Lett. 2010, 20, 7205-7211.

6. Rice, S.; Mason, H.D.; Whitehead, S.A. Phytoestrogens and their low dose combinations inhibit mRNA expression and activity of aromatase in human granulosa-luteal cells. J. Steroid Biochem. Mol. Biol. 2006, 101, 216-225.

7. Dube, P.N.; Mokale, S.N. Design and Synthesis of Some Novel Estrogen Receptor Modulators as Anti-Breast Cancer Agents: In Vitro \& In Vivo Screening, Docking Analysis. Anti-Cancer Agents Med. Chem. 2016, 16, 1461-1467.

8. Mokale, S.N.; Dube, P.N.; Bhavale, S.A.; Sayed, I.; Begum, A.; Nevase, M.C.; Shelke, V.R.; Mujaheed, A. Synthesis, in-vitro screening, and docking analysis of novel pyrrolidine and piperidine-substituted ethoxy chalcone as anticancer agents. Med. Chem. Res. 2015, 24, 1842-1856.

9. Dube, P.N.; Bule, S.S.; Mokale, S.N.; Kumbhare, M.R.; Dighe, P.R.; Ushir, Y.V. Synthesis and Biologic Evaluation of Substituted 5-methyl-2-phenyl-1H-pyrazol-3(2H)-one Derivatives as Selective COX-2 Inhibitors: Molecular Docking Study. Chem. Biol. Drug Des. 2014, 84, 409-419.

(C) 2019 by the authors. Licensee MDPI, Basel, Switzerland. This article is an open access article distributed under the terms and conditions of the Creative Commons Attribution (CC BY) license (http://creativecommons.org/licenses/by/4.0/). 